\title{
Attitude Angle Estimation of Space Probe through Wide-Field Integration of Optic Flow
}

\author{
By Hirofumi SAKАмото ${ }^{1)}$ and Shinji НокАмото ${ }^{1)}$ \\ Department of Aeronautics and Astronautics, Kyushu University, Fukuoka, Japan
}

(Received June 24th, 2013)

\begin{abstract}
This paper applies Wide-Field integration (WFI) of optic flow to the state estimation of a space probe. By utilizing nonlinear forms for optic flow instead of linearized expressions used in a standard method, this study enables the estimation of the probe's attitude angles as well as its velocity components. First, this paper describes the estimation principle of a new WFI method for three-dimensional motion of a space probe. Then, numerical simulations are conducted to verify the effectiveness of the proposed method. Through the simulation results, this paper discusses the effects of the following three factors on the estimation accuracy: resolution of optical images, roughness of target surface, and noise included in optic flow.
\end{abstract}

Key Words: Optic Flow, Wide-Field Integration, State Estimation, Autonomous System

Nomenclature

$\begin{array}{ll}d & : \text { distance measured by LRF } \\ F_{n} & : \text { sensitivity function } \\ J & : \text { performance index } \\ (p, q, r) & : \text { components of } \omega \\ \dot{\boldsymbol{Q}} & : \text { optic flow } \\ (u, v, w) & : \text { components of } \boldsymbol{v} \\ \boldsymbol{v} & : \text { translational velocity } \\ y_{n} & : \text { measurement output of WFI } \\ z & : \text { altitude } \\ (\phi, \theta, \psi) & : \text { attitude angles } \\ \mu & : \text { nearness function } \\ \omega & : \text { angular rate }\end{array}$

Subscripts

$b$

$n$

$t$

Accent

$\hat{*}$

$\widetilde{*}$

$* \dagger$

\section{Introduction}

These days, planet or asteroid exploration missions are planned in many countries. This indicates that the importance of space exploration missions have been widely recognized not only for space science but for technical view points, e.g. space observatory, in-situ resource utilization, and so on. In order to obtain precise information on a celestial body, operations in close proximity or landing missions on the target body are required. In Japan, an unmanned space probe Hayabusa succeeded in collecting the samples of an asteroid named Itokawa and in returning to Earth.
In close proximity operation or landing mission, space probes are required to know their states autonomously for obstacle avoidance or soft landing missions on a celestial body. This is because the communication delay between the probe and Earth becomes large especially in deep space missions. Thus for the success in deep space missions, probes must estimate its states with high accuracy even in unknown environments. On the other hand, the weight and the computational ability of deep space probes are strictly limited.

Wide-Field Integration (WFI) of optic flow is a state estimation method inspired by insect-vision systems. The WFI of optic flow can be implemented on a small vehicle with few computations, and still it can show good robustness even in unknown environments. Thus WFI of optic flow has been applied for speed control or collision avoidance in unknown environments: ${ }^{1-5)}$ typical applications are corridor-navigation, level flight with a constant speed, hovering control of vehicles, etc. In Ref. 6), our group has discussed how to apply the method to a space probe descending towards an asteroid. However, these works use a standard WFI of optic flow; in the standard method, linearized expressions are used for optic flow. Consequently, estimated state variables are limited to the vehicle's translational and angular velocities, although vehicle's position and attitude variables influence the optic flow. Furthermore, the standard method needs other sensors to obtain information on the attitude and position variables.

Contrary to those, this study utilizes nonlinear expressions for WFI of optic flow and shows that estimation of the attitude angles of a space probe is possible. In this paper the space probe is in the proximity of a celestial body, and its three-dimensional motion is treated. Furthermore to focus on the estimation of attitude angles, the probe is assumed to equip with a laser range finder (LRF) to measure a distance to a point on the asteroid surface. First this paper explains the principle of the state estimation and shows the algorithm of the proposed WFI method. Then, numerical simulations are 


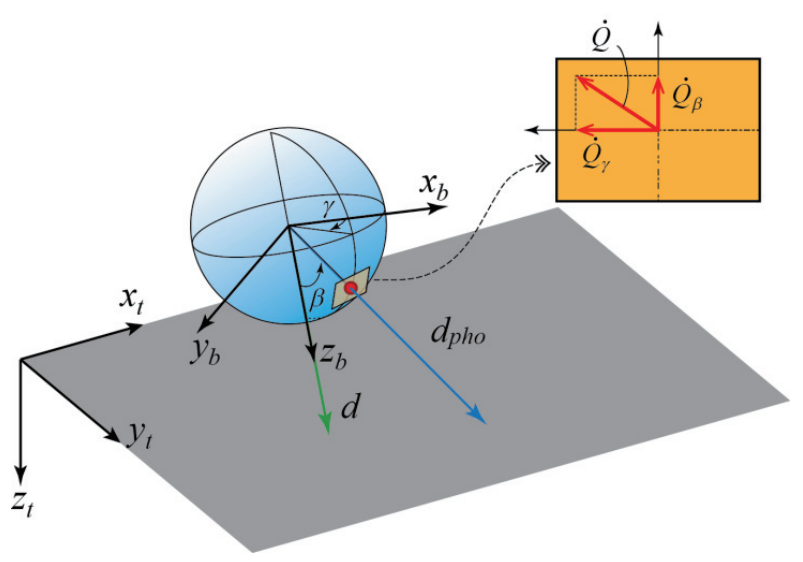

Fig. 1. Optic flow obtained on a spherical image plane.

conducted to verify the effectiveness of the proposed method. Through the simulation results, this paper discusses the effects of the following three factors on the estimation accuracy: resolution of optical images, roughness of target surface, and noise included in optic flow.

\section{Optic Flow and Wide-Field Integration}

\subsection{Optic flow configuration}

For the state estimation of a space probe in proximity to a target surface, let us consider Fig. 1 showing the configuration of the problem. In this figure, the subscript $b$ and $t$ denote body-fixed and target-fixed reference frames, respectively. Optic flow is measured with photoreceptors on a spherical image plane of the probe, and the photoreceptors' positions are expressed with two angles in Fig. 1: the azimuth angle $\gamma$ and the elevation angle $\beta$. The marker $\boldsymbol{Q}$ on the image plane is written with the distance vector $\boldsymbol{d}_{p h o}$ to a point on the target surface as follows:

$$
\boldsymbol{Q}=\frac{\boldsymbol{d}_{p h o}}{\left|\boldsymbol{d}_{p h o}\right|} .
$$

As described before, in this study, two sensors are used for the state estimation. One is an image sensor for measuring optic flow $\dot{\boldsymbol{Q}}$. The other is a LRF for measuring the distance $d$ along the $z_{b}$ direction to a point on the target surface. Thus, the sensor's outputs are composed of $\dot{\boldsymbol{Q}}$ and $d$ in this paper.

\subsection{Optic flow model}

Generally, optic flow can be written with vectors defined in body-fixed frame as follows: ${ }^{7)}$

$$
\dot{\boldsymbol{Q}}=-\boldsymbol{\omega} \times \boldsymbol{Q}-\mu\{\boldsymbol{v}-\langle\boldsymbol{v}, \boldsymbol{Q}\rangle \boldsymbol{Q}\},
$$

where $\langle *, *\rangle$ denotes an inner product, $\boldsymbol{\omega}=(p, q, r)$ is angular rate, and $\boldsymbol{v}=(u, v, w)$ is translational velocity of the probe. These velocity components are expressed in the body-fixed frame. The nearness function $\mu(\gamma, \beta, q)$ is defined as the inverse of $\left|\boldsymbol{d}_{p h o}\right|$, and thus it depends on the photoreceptor's position and the probe's pose $\boldsymbol{q}=(x, y, z, \phi, \theta, \psi)$. Here, the attitude angles $(\phi, \theta, \psi)$ are defined by the 3-2-1 Euler angles. Then the $\gamma$ and $\beta$ components of optic flow can be written as

$$
\begin{gathered}
\dot{Q}_{\gamma}(\gamma, \beta, \boldsymbol{q})=p \cos \beta \cos \gamma+q \cos \beta \sin \gamma \\
-r \sin \beta+\mu(u \sin \gamma-v \cos \gamma), \\
\dot{Q}_{\beta}(\gamma, \beta, \boldsymbol{q})=p \sin \gamma-q \cos \gamma \\
+\mu(-u \cos \beta \cos \gamma-v \cos \beta \sin \gamma+w \sin \beta) .
\end{gathered}
$$

\subsection{Wide-Field Integration}

In the WFI of optic flow, the measurement outputs $y_{n}$ are calculated through the integration of the inner product between $\dot{Q}$ and sensitivity functions $F_{n}$ as follows:

$$
y_{n}=\int \dot{Q}(\gamma, \beta, \boldsymbol{q}) \cdot F_{n}(\gamma, \beta) d \Omega,
$$

where $d \Omega=d \gamma \cdot d \beta \cdot \sin \beta$ is a solid angle on the image sphere, and spherical harmonics are used for $F_{n}$ in this study. The subscript $n \in\{1,2,3, \cdots, M\}$ indicates the mode number of the spherical harmonics.

Although optic flow $\dot{\boldsymbol{Q}}$ can be defined continuously over the image sphere, real optic flow is obtained with the photoreceptors discretely. Thus, this study approximates the output $y_{n}$ for the WFI of optic flow with Riemann sum as ${ }^{8)}$

$$
\tilde{y}_{n}=\sum_{k=1}^{N} \dot{\boldsymbol{Q}}\left(\gamma_{k}, \beta_{k}, \boldsymbol{q}\right) \cdot F_{n}\left(\gamma_{k}, \beta_{k}\right) \Delta \Omega,
$$

where * denotes the approximated value. Then, the approximated outputs combined from $n=1$ to $n=M$ can be expressed as

$$
\tilde{\boldsymbol{y}}=\left(\left[\begin{array}{ccc}
F_{1}\left(\gamma_{1}, \beta_{1}\right) & \cdots & F_{1}\left(\gamma_{N}, \beta_{N}\right) \\
\vdots & \ddots & \vdots \\
F_{M}\left(\gamma_{1}, \beta_{1}\right) & \cdots & F_{M}\left(\gamma_{N}, \beta_{N}\right)
\end{array}\right]\left[\begin{array}{c}
\dot{\boldsymbol{Q}}\left(\gamma_{1}, \beta_{1}, \boldsymbol{q}\right) \\
\vdots \\
\dot{\boldsymbol{Q}}\left(\gamma_{N}, \beta_{N}, \boldsymbol{q}\right)
\end{array}\right]\right) \Delta \Omega .
$$

Thus, by defining the following matrices

$$
\begin{aligned}
& {[F]=\left[\begin{array}{ccc}
F_{1}\left(\gamma_{1}, \beta_{1}\right) & \cdots & F_{1}\left(\gamma_{N}, \beta_{N}\right) \\
\vdots & \ddots & \vdots \\
F_{M}\left(\gamma_{1}, \beta_{1}\right) & \cdots & F_{M}\left(\gamma_{N}, \beta_{N}\right)
\end{array}\right],} \\
& {\left[\dot{Q}_{\gamma}\right]=\left[\begin{array}{lll}
\dot{Q}_{\gamma}\left(\gamma_{1}, \beta_{1}, \boldsymbol{q}\right) & \cdots & \dot{Q}_{\gamma}\left(\gamma_{N}, \beta_{N}, \boldsymbol{q}\right)
\end{array}\right]^{T} \text {, }} \\
& {\left[\dot{Q}_{\beta}\right]=\left[\begin{array}{lll}
\dot{Q}_{\beta}\left(\gamma_{1}, \beta_{1}, \boldsymbol{q}\right) & \cdots & \dot{Q}_{\beta}\left(\gamma_{N}, \beta_{N}, \boldsymbol{q}\right)
\end{array}\right]^{T},}
\end{aligned}
$$

this study expresses the $\gamma$ and $\beta$ components of $\tilde{\boldsymbol{y}}$ as follows:

$$
\begin{aligned}
& \tilde{\boldsymbol{y}}_{\gamma}=[F]\left[\dot{Q}_{\gamma}\right] \Delta \Omega, \\
& \tilde{\boldsymbol{y}}_{\beta}=[F]\left[\dot{Q}_{\beta}\right] \Delta \Omega .
\end{aligned}
$$

\subsection{State variables estimation}

In real environments, the nearness function $\mu(\gamma, \beta, q)$ depends on the roughness of target surfaces. However, this study assumes in the estimation process that the target surface is flat, because getting precise information on touchdown area in advance is usually very difficult. For the flat surface, the nearness function is described according to the attitude angles as

$$
\begin{aligned}
\mu(\gamma, \beta, \boldsymbol{q}) & =\frac{1}{z}(-\sin \beta \cos \gamma \sin \theta \\
& +\sin \beta \sin \gamma \sin \phi \cos \theta+\cos \beta \cos \phi \cos \theta) .
\end{aligned}
$$

Note that the yaw angle $\psi$ does not appear in this equation under the assumption of a flat surface.

Substituting Eq. (13) into Eqs. (3) and (4) indicates that optic flow is a function of two attitude angles $(\phi, \theta)$, an altitude 
Table 1. The coefficient matrix $\boldsymbol{C}_{a}(\phi, \theta)$

\begin{tabular}{|c|cccccc|}
\hline direction (a) & component of matrix \\
\hline$\gamma$ & $\sum_{k=1}^{N}\left[\begin{array}{ccccccc}\lambda(\phi, \theta) \sin \gamma_{k} \cdot F_{1} & -\lambda(\phi, \theta) \cos \gamma_{k} \cdot F_{1} & 0 & \cos \beta_{k} \cos \gamma_{k} \cdot F_{1} & \cos \beta_{k} \sin \gamma_{k} \cdot F_{1} & -\sin \beta_{k} \cdot F_{1} \\
\vdots & \vdots & \vdots & \vdots & \vdots & \vdots \\
\lambda(\phi, \theta) \sin \gamma_{k} \cdot F_{M} & -\lambda(\phi, \theta) \cos \gamma_{k} \cdot F_{M} & 0 & \cos \beta_{k} \cos \gamma_{k} \cdot F_{M} & \cos \beta_{k} \sin \gamma_{k} \cdot F_{M} & -\sin \beta_{k} \cdot F_{M}\end{array}\right]$ \\
\hline \multirow{2}{*}{$\beta$} & $\sum_{k=1}^{N}\left[\begin{array}{ccccccc}-\lambda(\phi, \theta) \cos \beta_{k} \cos \gamma_{k} \cdot F_{1} & -\lambda(\phi, \theta) \cos \beta_{k} \sin \gamma_{k} \cdot F_{1} & \lambda(\phi, \theta) \sin \beta_{k} \cdot F_{1} & \sin \gamma_{k} \cdot F_{1} & \cos \gamma_{k} \cdot F_{1} & 0 \\
\vdots & \vdots & \vdots & \vdots & \vdots \\
-\lambda(\phi, \theta) \cos \beta_{k} \cos \gamma_{k} \cdot F_{M} & -\lambda(\phi, \theta) \cos \beta_{k} \sin \gamma_{k} \cdot F_{M} & \lambda(\phi, \theta) \sin \beta_{k} \cdot F_{M} & \sin \gamma_{k} \cdot F_{M} & \cos \gamma_{k} \cdot F_{M} & 0\end{array}\right]$ \\
\hline
\end{tabular}

where $\lambda(\phi, \theta)=-\sin \beta \cos \gamma \sin \theta+\sin \beta \sin \gamma \sin \phi \cos \theta+\cos \beta \cos \phi \cos \theta$

$z$, three translational velocities $(u, v, w)$, and three angular rates $(p, q, r)$. Note that the translational velocities $(u, v, w)$ in optic flow always appear as a form divided by the altitude $z$. This means that the altitude and the translational velocities cannot be separated. Thus, this paper defines the following vector $\boldsymbol{X}$ as the velocity components of the probe.

$$
\begin{gathered}
\boldsymbol{X}=\left[\begin{array}{llllll}
u / z & v / z & w / z & p & q & r
\end{array}\right]^{T} \\
\square\left[\begin{array}{llllll}
U & V & W & p & q & r
\end{array}\right]^{T} .
\end{gathered}
$$

Then the $\gamma$ and $\beta$ components of the approximated outputs in Eqs. (11) and (12) are rewritten in the following form

$$
\tilde{\boldsymbol{y}}_{a}=\boldsymbol{C}_{a}(\phi, \theta) \boldsymbol{X} \quad(a=\gamma, \beta) .
$$

The components of the matrix $\boldsymbol{C}_{a}(\phi, \theta)$ are expressed in Table 1 . The vector $\boldsymbol{X}$ can be expressed by using the pseudo inverse matrix of $\boldsymbol{C}_{a}$ as

$$
\boldsymbol{X}=\boldsymbol{C}_{a}^{\dagger}(\phi, \theta) \tilde{\boldsymbol{y}}_{a} .
$$

Substituting Eq. (16) into Eq. (15) becomes

$$
\tilde{\boldsymbol{y}}_{a}=\boldsymbol{C}_{a}(\phi, \theta) \boldsymbol{C}_{a}^{\dagger}(\phi, \theta) \tilde{\boldsymbol{y}}_{a} .
$$

This equation should be correct for true attitude angles, if the quantization error is negligible. Thus, this relation can be used for estimation of the attitude angles.

This paper expresses the difference between the both sides of Eq. (17) as

$$
\boldsymbol{\delta}_{a}(\phi, \theta)=\tilde{\boldsymbol{y}}_{a}-\boldsymbol{C}_{a}(\phi, \theta) \boldsymbol{C}_{a}^{\dagger}(\phi, \theta) \tilde{\boldsymbol{y}}_{a},
$$

and defines the performance index to evaluate the attitude angle error as follows:

$$
J(\phi, \theta)=\ln \left[\boldsymbol{\delta}_{\gamma}^{T}(\phi, \theta) \boldsymbol{\delta}_{\gamma}(\phi, \theta)+\boldsymbol{\delta}_{\beta}^{T}(\phi, \theta) \boldsymbol{\delta}_{\beta}(\phi, \theta)\right] .
$$

Hereafter the estimated attitude angles which minimize the above performance index are expressed with hat as $\hat{*}$.

Since the translational velocities always appear as a form divided by the altitude, a LRF is used to specify the altitude information in this paper. When the LRF is placed along the $z_{b}$ direction, the altitude is calculated as follows:

$$
\hat{z}=d \cos \hat{\phi} \cos \hat{\theta} \text {. }
$$

Then, the translational and rotational velocities are evaluated from Eq. (16) by using the estimated altitude. It should be noted in Eq. (16) that the first and fifth columns of $\boldsymbol{C}_{\gamma}(\phi, \theta)$ and the second and fourth columns of $\boldsymbol{C}_{\beta}(\phi, \theta)$ become close to a zero-vector in the vicinity of $(\phi, \theta)=(0,0)$. This means that the first and fifth components of $\boldsymbol{X}$ should be estimated by using $\boldsymbol{C}_{\beta}$ and that the second and fourth

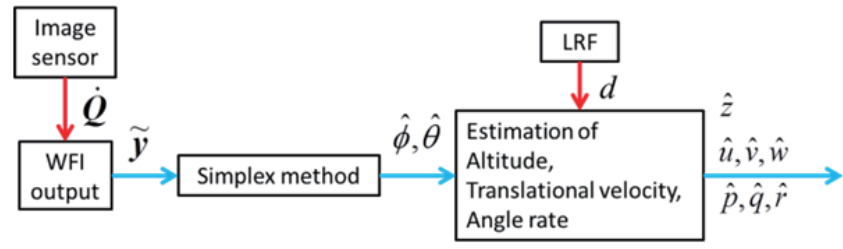

Fig. 2. The flowchart of the algorithm.

components should be evaluated by using $\boldsymbol{C}_{\gamma}$ as follows:

$$
\begin{gathered}
\hat{u}=\hat{U}_{\beta} \cdot \hat{z}, \quad \hat{v}=\hat{V}_{\gamma} \cdot \hat{z}, \quad \hat{w}=\hat{W}_{\beta} \cdot \hat{z}, \\
\hat{p}=\hat{p}_{\gamma}, \quad \hat{q}=\hat{q}_{\beta}, \quad \hat{r}=\hat{r}_{\gamma} .
\end{gathered}
$$

\subsection{Estimation through a simplex method}

Fig. 2 shows the flowchart of the estimation process for the state variables used in this paper. First, optic flow $\dot{\boldsymbol{Q}}$ is obtained from image sensor's outputs, and from the optic flow the WFI output $\tilde{\boldsymbol{y}}$ is approximated. Then, the probable attitude angles are searched through a simplex method.

Simplex methods are frequently used in linearized programing for nonlinear problems. This study uses the following performance index for the linearized problem to search the probable attitude angles.

$$
J_{\text {simplex }}(\phi, \theta)=\kappa J^{-}\left(\phi^{-}, \theta^{-}\right)+(1-\kappa) J(\phi, \theta),
$$

where the superscript ' - ' indicates that the value is calculated at the previous time step. This is based on an assumption that the attitude angles are almost the same as the previous ones after a brief time interval. Thus, $J^{-}$is the performance index calculated in Eq. (19) by using the previous $\left(\phi^{-}, \theta^{-}\right)$. The value of $\kappa$ means the ratio between $J^{-}$and $J$.

From the estimated attitude angles and the LRF output, the altitude is calculated from Eq. (20), and finally the translational and rotational velocities are estimated in Eq. (21).

\section{Numerical Simulations}

Table 2. Initial states of a space probe.

\begin{tabular}{|c|c|}
\hline Variables & Value \\
\hline \hline$(\phi, \theta)[\mathrm{deg}]$ & $(5.83,-7.90)$ \\
\hline $\mathrm{z}[\mathrm{m}]$ & -50 \\
\hline$(u, v, w)[\mathrm{m} / \mathrm{s}]$ & $(-0.00068,-.0 .0133,-0.0287)$ \\
\hline$(p, q, r)[\mathrm{deg} / \mathrm{s}]$ & $(-0.180,0100,-0.0499)$ \\
\hline
\end{tabular}




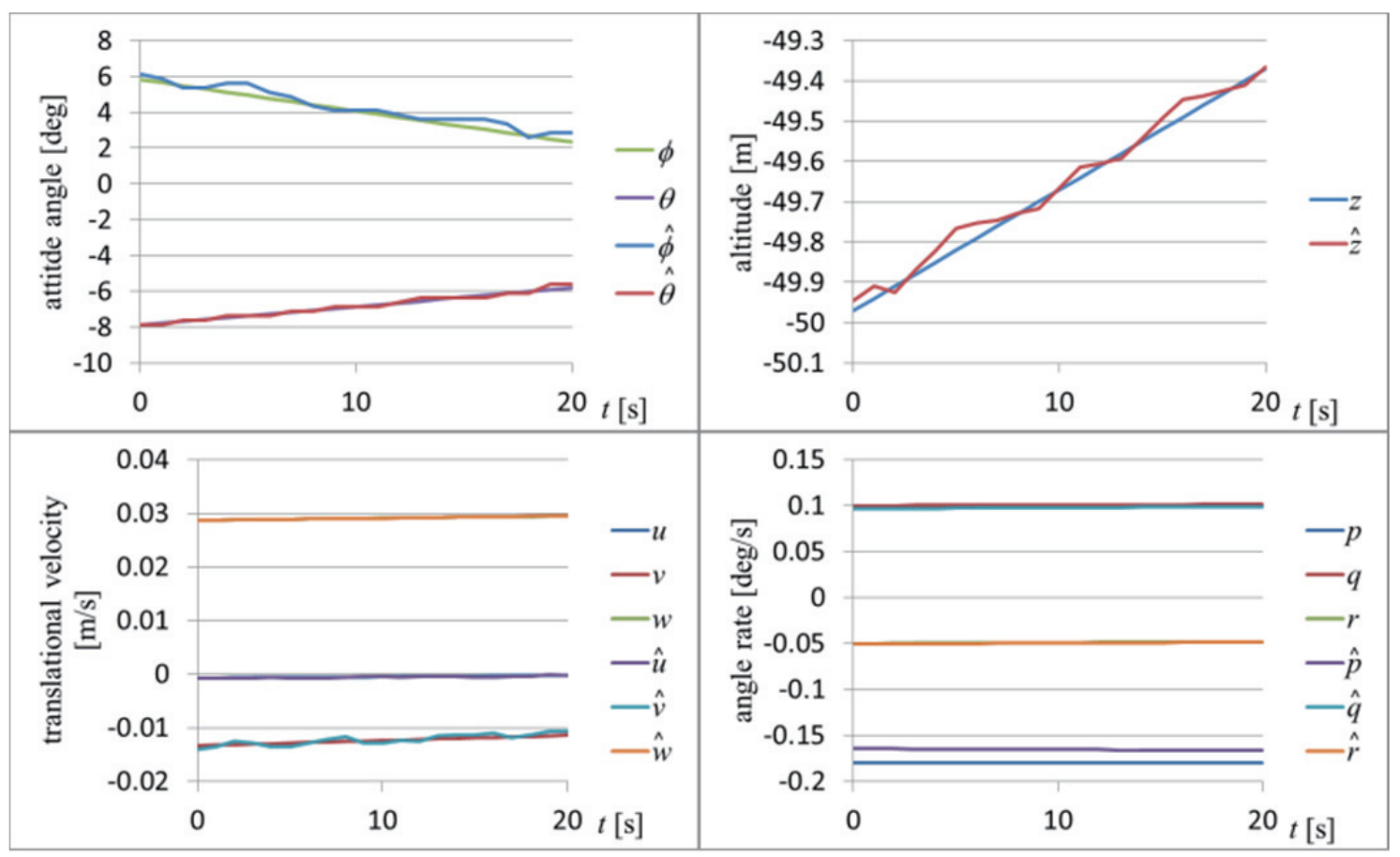

Fig. 3. Estimation results of a probe's state variables obtained from a proposed WFI method for a flat plane.

Table 2 indicates the initial states of a space probe in proximity to a target surface used in numerical simulations. Since no control maneuver is applied to the probe, its attitude and altitude parameters change continuously with constant rates. In the simulations, optic flow is assumed to be obtained every second with onboard image sensors.

\subsection{Estimation for a flat surface}

First, a flat plane is used as a target surface to verify the basic performance of the proposed WFI method. In the estimation process, the probable attitude angles are sought through a simplex algorithm by using Eq. (22). The constant value $\kappa$ has been decided beforehand so that the estimation error of the attitude angles becomes minimal through 100 cases of Monte Carlo simulations. In these preliminary simulations, the initial attitude angles are randomly selected within $|\phi|,|\theta| \leq 15[\mathrm{deg}]$, because in most scenarios $\phi=\theta=0$ [deg] is used as reference attitude angles in proximity missions.

Fig. 3 shows the estimation results of the states. It indicates that the proposed method can estimate the attitude angles of the probe as well as the velocity components. Even the maximum error of the estimated attitude angle is smaller than $0.6[\mathrm{deg}]$ in this case. Furthermore, the altitude is evaluated from a LRF with a good accuracy.

It is easily anticipated that the estimation accuracy depends on the resolution of images used in the WFI method. Thus, the relationship between the resolution of an image sensor and the estimation error is examined. To specify the resolution of images, this paper defines an angle $\alpha[\mathrm{deg}]$ indicating a constant interval between photoreceptors in both $\gamma$ and $\beta$ directions. Fig. 4 shows the result. It indicates that the estimation accuracy becomes higher according to the resolution of images. This result, however, implies that the expected estimation accuracy should be decided as a tradeoff

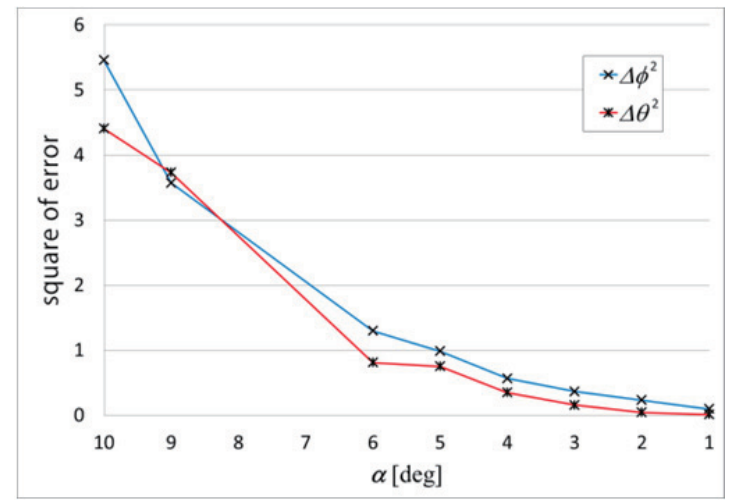

Fig. 4. Effect of a sensor resolution on estimation accuracy.

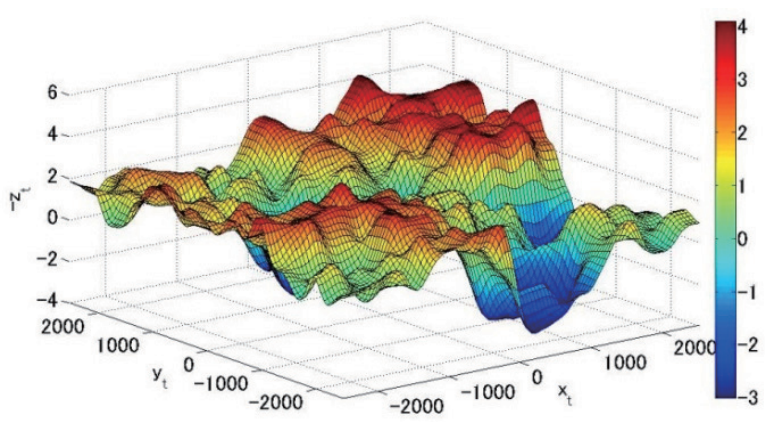

Fig. 5. A rough surface model used as an assumed target surface. 


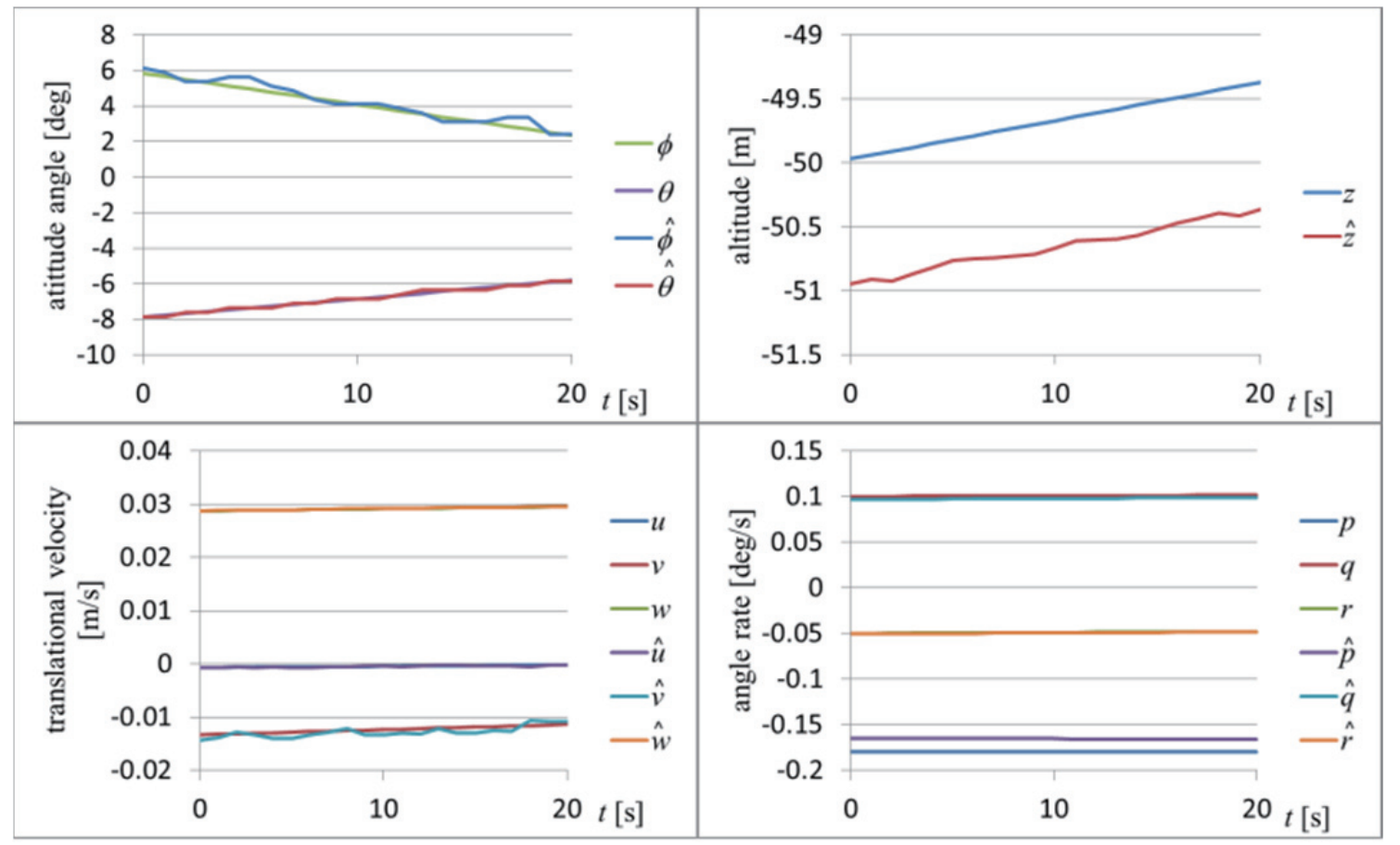

Fig. 6. Estimation results of a probe's state variables for a rough surface.

with a required computational cost. This is because, for higher resolution images, the computational time and required memory become larger. (Fig. 3 has been calculated for $\alpha=1$ [deg].)

\subsection{Estimation for a rough surface}

The previous subsection indicates that the proposed WFI method is effective for a flat surface. While the standard WFI of optic flow method is regarded as robust for unknown environments due to the integration process over a wide field-of-view. Thus, the robustness of the new WFI method on rough terrain is examined in numerical simulations.

Fig. 5 shows a rough surface model used in the examination. This surface is generated by using random numbers and subsequently smoothed through a Gaussian filter. The highest points in this surface are limited to $5[\mathrm{~m}]$.

Fig. 6 shows the estimation results of the states for the rough surface. The simulation conditions are same as those for Fig. 3, except the surface roughness. This result shows that the attitude angles can be estimated successfully even for the rough surface. Moreover, the estimation accuracy is almost kept even for the rough surface. Thus, the new WFI method is expected to have a same level of robustness as the standard method. On the other hand, the estimated altitude has around 1 $[\mathrm{m}]$ error, but it is rather smaller compared with the roughness range of the surface.

\subsection{Effect of "noise" on the estimation accuracy}

In the previous examinations, it is assumed that optic flow $\dot{\boldsymbol{Q}}$ is obtained with photoreceptors placed at equal intervals in any directions and the obtained optic flow is "perfectly correct". However, in a realistic situation, several cameras are probably substituted to obtain optic flow on a spherical image plane. This substitution generates errors on the photoreceptor positions. For examples, Fig. 7(top) shows a probe with six
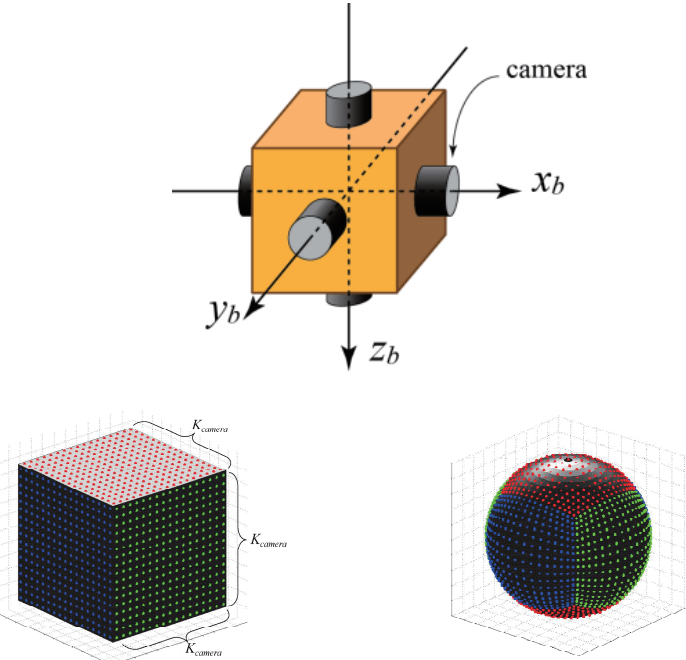

Fig. 7. Six camera systems on a space probe (top), its photoreceptors distributed on a cube (bottom left), and the converted ones on a sphere (bottom right).

camera systems. For this system, the positions of supposed photoreceptors are distributed on a cube (bottom left), and finally they are mapped on a sphere (bottom right). Consequently, the converted positions of the photoreceptors are not placed at equal intervals. However, the WFI method for uneven positions of photoreceptors makes the estimation calculation quite complicated. Thus, this study assumes equal intervals for the photoreceptors, and treats the error between even and uneven positions as "noise".

On the other hand, optic flow may be contaminated with "noise" in its generation process. To evaluate the effect of the noise, it is assumed that optic flow is generated through a gradient method and that the derivative values of the 

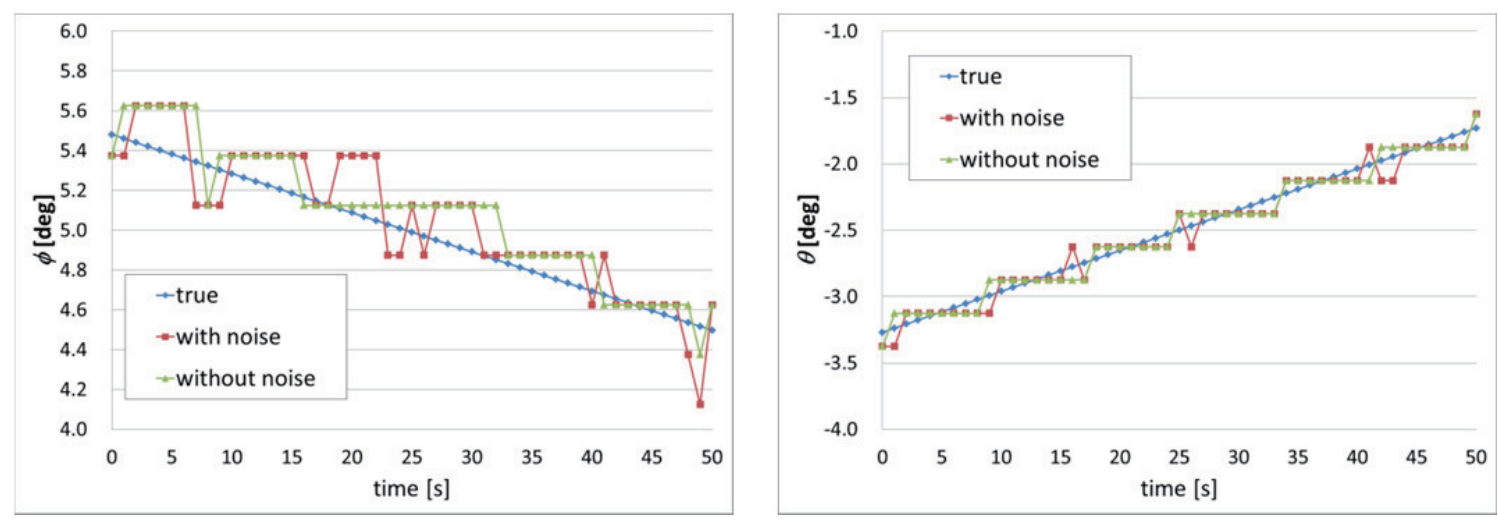

Fig. 8. Estimation results of a probe's attitude angles with / without noise.

brightness information on sensor images include noise. In this paper, the covariance values of the noise are set as 2.0 for the two directions on the image plane and 0.3 for the time axis, from an experimental estimation. ${ }^{9)}$ Then, the covariance matrix of the noise model can be calculated as the Cramer-Rao lower bound. ${ }^{10)}$

Furthermore, the output of a LRF for the altitude measurement also includes a noise. The noise model evaluated for LRF-S1, which was used in the Hayabusa spacecraft, is used as a LRF noise and included in the simulation.

Fig. 8 shows a typical result for the attitude angles estimations with and without the noises. The result indicates that the attitude angles can be estimated with almost the same accuracy even when the noises are included to the sensor outputs.

Furthermore, to evaluate the effect of the noise on the estimation accuracy statistically, 100 times of simulations are conducted for different initial states. Table 3 denotes the root mean square (RMS) of the estimation error for each state variable. This table indicates that the estimation accuracy for the attitude angles and the rotational velocities is not much deteriorated when the noises are added, while the estimation errors for the altitude and the translational velocities become large. This is caused by that the translational velocities are evaluated by using the estimated altitude data as shown in Eq. (21). Thus, those variables are directly affected by the noise added in the LRF. It should be noted that, although the altitude and the translational velocities are sensitive for noises, the error level is still small. Therefore the result implies that the proposed WFI method is effective when noises are included in the sensor outputs.

\section{Conclusion}

This paper has proposed a new WFI method which can estimate the attitude angles of a space probe. For this purpose, nonlinear expressions for optic flow in three-dimensional motion are utilized and integrated over a wide field-of-view area. The numerical simulations shown in this paper verify that the proposed method is effective for the estimation of the attitude angles as well as the velocity components. The effects of image resolutions, surface roughness, and noises on the
Table 3. RMS of the estimation errors.

\begin{tabular}{|c|c|c|c|c|c|c|}
\hline & $\phi$ & $\theta$ & $z$ & & & \\
\hline with noise & 3.78.E-01 & 1.27.E-01 & 4.82.E-01 & & & \\
\hline without noise & 3.23.E-01 & 1.18.E-01 & 2.64.E-02 & & & \\
\hline & $u$ & $v$ & $w$ & $p$ & $q$ & $r$ \\
\hline with noise & 1.38.E-04 & 1.51.E-02 & 2.13.E-04 & 6.58.E-03 & 2.92.E-03 & 2.97.E-04 \\
\hline without noise & 6.68.E-05 & 4.55.E-03 & 2.05.E-05 & 5.88.E-03 & 3.33.E-03 & 3.15.E-04 \\
\hline
\end{tabular}

estimation accuracy have been also investigated numerically. Those results show that the proposed method is effective and robust for rough surfaces and sensor noises.

\section{References}

1) Humbert, J. S., Murray, R. M. and Dickinson M. H.: A Control-Oriented Analysis of Bio-inspired Visuomotor Convergence, Proceedings of the IEEE Conference on Decision and Control, 2005, pp. 245-250.

2) Humbert, J. S. and Frye, M. A.: Extracting Behaviorally Relevant Retinal Image Motion Cues via Wide-Field Integration, Proceedings of the American Control Conference, 2007, pp.2724-2729.

3) Epstein, M., Waydo, S., et al.: Biologically Inspired Feedback Design for Drosophila Flight, Proceedings of the American Control Conference, 2007, pp.3395-3401.

4) Hyslop, A. M., Krapp, H. G. and Humbert J. S.: Control theoretic interpretation of directional motion preferences in optic flow processing interneurous, Journal of Biological Cybernetics, 103 (2010), pp.353-364.

5) Hyslop, A. M. and Humbert, J. S.: Autonomous Navigation in Three-Dimensional Urban Environments Using Wide-Field Integration of Optic Flow, Journal of Guidance, Control, and Dynamics, 33 (2010), pp.147-159.

6) Shoemaker, M. A. and Hokamoto, S.: Application of Wide-Field Integration of Optic Flow to Proximity Operations and Landing for Space Exploration Missions, Advances in the Astronautical Sciences, 142 (2011), pp.23-36.

7) Koenderink, J. J. and van Doorn, A. J.: Facts on Optic Flow, Journal of Biological Cybernetics, 56 (1987), pp.247-254.

8) Shoemaker, M. A. and Hokamoto, S.: Comparison of Integrated and Nonintegrated Wide-Field Optic Flow for Vehicle Navigation, Journal of Guidance, Control, and Dynamics, 36 (2013), pp.710-720.

9) Kimura, K., Ohta, N. and Kanatani, K.: Optical Flow Detection Using a New Uncertainty Model of Gradient Constraint, JPSJ SIG Notes, (1996), pp.37-42 (in Japanese).

10) Kanatani, K.: Statistical Optimization for Geometric Computing. Theory and Practice, Elsevier Science, 1996. 